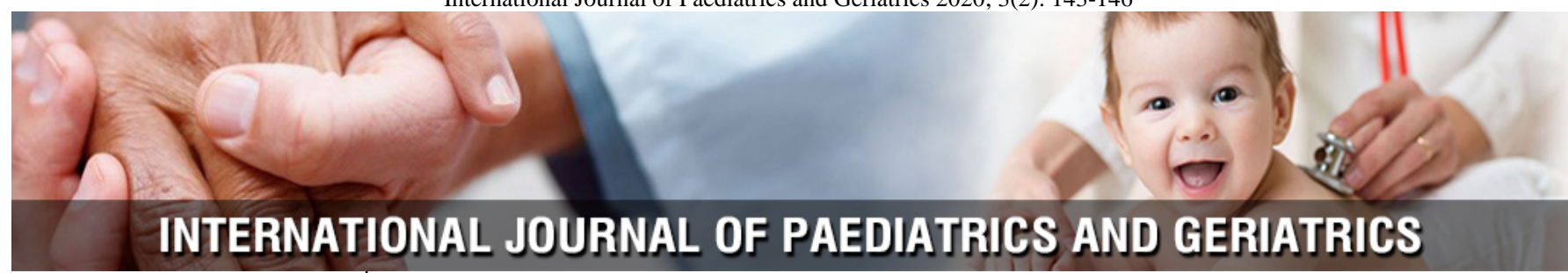

P-ISSN: 2664-3685

E-ISSN: $2664-3693$

IJPG 2020; 3(2): 143-146

Received: 09-05-2020

Accepted: 13-06-2020

Gonesh N Mevundi SNCU Nodal Officer, Department of Paediatrics, District Hospital, Koppal,

Karnataka, India

Harsha Sangolgikar Senior Resident Senior Resident, Department of Paediatrics, KIMS, Koppal, Karnataka, India

\section{Organism involved and clinical presentation in proven neonatal sepsis affecting platelet indices}

\author{
Gonesh N Mevundi and Harsha Sangolgikar
}

DOI: https://doi.org/10.33545/26643685.2020.v3.i2c.107

\begin{abstract}
Background: Sepsis is a common complication in the neonatal intensive care unit and is a major cause of neonatal mortality.

Objective: To identify organism involved in proven neonatal sepsis affecting platelet indices.

Setting: The study was carried out over a period of one and half year from December 2015 to July 2017 at Sangmeshwar and Basaveshwar Hospital attached to M.R. Medical College, KALABURAGI. 100 cases were considered for this study after proper screening for CBC, platelet count and their indices like mean platelet volume, platelet distribution width and CRP and blood culture in neonates admitted in our NICU with proven sepsis

Result: A total of 100 neonates with blood culture positive for bacterial cases were considered for the study. Early onset septicaemia (59\%) was more common than late onset septicaemia (41\%). Out of 100 cases $57 \%$ cases had growth of gram negative organisms, $40 \%$ had growth of gram positive organisms and 3\% had growth of fungal. Tachypnea (27\%), Lethargy (20\%) and refusal of feeds (8\%) were the commonest clinical presentation followed by, Fever (6\%), convulsions (5\%) and jaundice (5\%). 60\% neonates had thrombocytopenia of varying severity. Staphylococcus aureus was the most common organism associated with thrombocytopenia (43.3\%).

Conclusion: It was concluded that neonatal sepsis was common in males. Gram positive organisms were the predominant causative agents of septicaemia $40 \%$ as compared to gram negative organisms $57 \%$ and fungal sepsis $3 \%$. Staphylococcus aureus was the commonest organism responsible for thrombocytopenia.
\end{abstract}

Keywords: Neonatal sepsis, thrombocytopenia, staphylococcus aureus, clinical presentation, lethargy

\section{Introduction}

Neonatal septicemia is a clinical syndrome characterized by signs and symptoms of infection with or without accompanying bacteremia in the first month of life. ${ }^{[1]}$. It is caused by various organisms invading the blood stream, which may be by bacterial, viral, fungal and protozoal infections. It is characterized by positive blood culture, thrombocytopenia and elevated C-reactive protein. Septic shock is the most dangerous complication of septicaemia ${ }^{[1]}$ In contrast, only $2 \%$ of the neonates are thrombocytopenic at birth with Severe Thrombocytopenia (platelet count $<50,000 / \mu \mathrm{L}$ ) occurring in less than $3 / 1000$ term infants ${ }^{\text {[2] }}$ Megakaryopoiesis and thrombopoiesis and platelet physiology in the fetus and neonate:

Platelets are small anucleate fragments that are formed from the cytoplasm of megakaryocytes and have a characteristic discoid shape ${ }^{[3]}$.

Megakaryopoesis include the production of megakaryocytes from stem cells, while thrombopoiesis is the production of platelets from megakaryocytes.

Platelet production begins in the yolk sac and, like the remainder of hematopoiesis shifts to the fetal liver and then to the marrow at the time of gestation ${ }^{[4]}$.

The most primitive progenitor cell that gives rise to megakaryocytic lineage cells is the multipotent progenitor, CFU-GEMM (Colony forming unit-granulocyte I erythrocyte/ monocyte/megakaryocyte) ${ }^{[5]}$. The most primitive progenitor cell committed exclusively to the megakaryocytic lineage is BFU-MK (Burst forming unit megakaryocyte) [5]. Its immediate mature progeny is CFU- MK, which is CD34, CD 41, c-mpl and HLA-DR positive $^{[5]}$. All the major megakaryoctyes progenitor and precursor cells have been identified in the fetus and the newborn ${ }^{[6]}$.

Hence this study was conducted to identify organism involved in proven neonatal sepsis affecting platelet indices. 


\section{Materials \& Methods}

The study subjects are all neonates admitted in Basaweshwar and Sangameshwar hospital NICU attached to M.R. Medical College and has proven sepsis.

Inclusion criteria: All neonates admitted in our NICU with proven sepsis.

\section{Exclusion criteria}

1. Causes of thrombocytopenia other than sepsis

2. Neonates whose parents or guardians did not agree to be a part of study.

The Prospective hospital based study was carried out over a period of one and half year from December 2015 to July 2017 at Sangmeshwar and Basaveshwar Hospital. attached to M.R.Medical College, Kalaburagi.

100 cases were considered for this study after proper screening for CBC, platelet count and their indices like mean platelet volume, platelet distribution width and CRP and blood culture in neonates admitted in our NICU with proven sepsis.

Volume of blood: The chance of growing an organism effectively increases following inoculation of $0.5 \mathrm{ml}$ venous blood in a pediatric blood culture bottle or $1 \mathrm{ml}$ in an adult blood culture bottle (if the pediatric bottle is not available). ${ }^{\text {[7] }}$ The anticoagulant recommended for the blood culture is sodium Polyanethol Sulfonate (SPS Liquiod) in concentration of $0.0025 \%$ to $0.003 \%$.

Methods of collection of blood: Collecting a blood sample for culture was carried out under strict aseptic conditions to avoid contamination.

Sterile gloves were worn prior to the procedure and a patch of skin approximately $5 \mathrm{~cm}$ in diameter over the proposed veni-puncture site was prepared. This area was cleaned thoroughly with alcohol followed by povidine-iodine followed again by alcohol. Application of povidine-iodine was done in concentric circles moving outwards from the centre to avoid contamination. The skin was allowed to dry for at least minute before sample is collected. Once blood was drawn and inoculated in to the appropriate media, it was immediately sent to the microbiology laboratory for incubation. Blood culture bottles or tubes were never inoculated when the medium was cold nor were they refrigerated after inoculation.

This technique measures the $\mathrm{CO}_{2}$ derived $\mathrm{pH}$ changes by a colometric sensor in the bottom of each bottle the sensor is separated from the broth medium by a membrane that is only permeable to $\mathrm{CO}_{2}$.

As organisms grow they release $\mathrm{CO}_{2}$ which diffuses across the membrane and is dissolved in water present in the matrix of the sensor. As $\mathrm{CO}_{2}$ is dissolved, free hydrogen ions are generated. These freely generated hydrogen ions cause a colour change in the sensor which is read by the instrument.

Culture techniques: BacT/ALERT automated blood culture system is used to determine the growth of the organism. This technique measures the $\mathrm{CO}_{2}$ derived $\mathrm{pH}$ changes by a colometric sensor in the bottom of each bottle. The sensor is separated from the broth medium by a membrane that is only permeable to $\mathrm{CO}_{2}$. As organisms grow they release $\mathrm{CO}_{2}$ which diffuses across the membrane and is dissolved in water present in the matrix of the sensor. As $\mathrm{CO}_{2}$ is dissolved, free hydrogen ions are generated.

These freely generated hydrogen ions cause a colour change in the sensor which is read by the instrument. Within 6-18 hours of incubation most bacteria responsible for a clinically significant disease are present in numbers large enough to give a positive signal.66 Quick screening methods like quantitative direct plating (QDP) by placing few drops of blood may be useful where bacteraemia is of high degree or in neonates. Other sophisticated techniques in rapid isolation of organisms are by the use of radio-labelled carbon (14C) and automated techniques are recommended by some. Blood culture reports were declared at 3-5 days of incubation period. Those babies with proven bacterial sepsis were included in the study and platelet counts, bleeding manifestations and causative organisms were noted. $2 \mathrm{ml}$ venous blood samples were taken in EDTA bulbs for platelet count analysis using automated analyser.

\section{Result}

The study reveals that, most patients 53 (53.0\%) presented within 24 hours of age, followed by $11(11.0 \%)$ patients who presented after 48 hours of age. The minimum age of a patient was 1 day (24 hours) and maximum age of a patient was 9 days. The Mean and SD of age of males was $74.51 \pm$ 64.52 hours and females was $71.03 \pm 53.49$ hours. Overall Mean age of all patients was $72.46 \pm 57.23$.

There was no statistically significant difference of age of patients among males and females $(\mathrm{P}>0.05)$.

The sex ratio of male to female in the study was observed to be $1.85: 1$ Table 1.

Table 1: Distribution of Patients according to age and sex

\begin{tabular}{|c|c|c|c|c|c|c|}
\hline \multirow{2}{*}{ Age in hours } & \multicolumn{2}{|c|}{ Males } & \multicolumn{2}{|c|}{ Females } & \multicolumn{2}{|c|}{ Total } \\
\hline & No. & $\%$ & No. & $\%$ & No. & $\%$ \\
\hline 24 hours & 33 & 50.7 & 20 & 57.1 & 53 & 53.0 \\
\hline 48 hours & 6 & 9.2 & 3 & 8.6 & 9 & 9.0 \\
\hline 72 hours & 7 & 10.8 & 4 & 11.4 & 11 & 11.0 \\
\hline 96 hours & 6 & 9.2 & 3 & 8.6 & 9 & 9.0 \\
\hline 120 hours & 5 & 7.7 & 2 & 5.7 & 7 & 7.0 \\
\hline$>120$ hours & 8 & 12.4 & 3 & 8.6 & 11 & 11.0 \\
\hline Total & 65 & 100.0 & 35 & 100.0 & 100 & 100.0 \\
\hline Mean \pm SD & \multicolumn{2}{|c|}{$74.51 \pm 64.52$} & \multicolumn{2}{|c|}{$71.03 \pm 53.49$} & \multicolumn{2}{|c|}{$72.46 \pm 57.23$} \\
\hline t-test value & \multicolumn{4}{|c|}{$t=0.78$} & \multicolumn{2}{|c|}{---} \\
\hline P-value \& Significance & \multicolumn{4}{|c|}{$4 \mathrm{NS}$} & \multicolumn{2}{|c|}{---} \\
\hline
\end{tabular}

The study reveals that, most of the organisms isolated were Gram-negative (57\%), followed by gram positive (40\%) and fungal (3\%). Table 2 
Table 2: Organism Causing Neonatal Sepsis

\begin{tabular}{|c|c|c|c|}
\hline Variable & Organisms & No. of patients & \% \\
\hline \multirow{3}{*}{ Gram-Positive 40 (40.0\%) } & Staph aureus & 37 & 37.0 \\
\cline { 2 - 4 } & CoNS & 2 & 2.0 \\
\cline { 2 - 4 } & Enterococcus & 1 & 1.0 \\
\hline \multirow{3}{*}{ Gram-Negative 57 (57.0\%) } & E coli & 24 & 24.0 \\
\cline { 2 - 4 } & Klebsiella & 24 & 24.0 \\
\cline { 2 - 4 } & Pseudomonas & 9 & 11.6 \\
\hline Fungal 3 (3.0\%) & Candida & 3 & 100.0 \\
\hline Total & -- & 100 & 2.0 \\
\hline
\end{tabular}

It was observed that, Intramural patients were 45 (45.0\%) and Extramural patients were 55(55.0\%). In our study sepsis due to patients born outside hospital were more than those inside the hospital. Of the extramural, patients born in private hospital were $29 \%$ followed by primary health centre (16\%), govt general hospital (9\%) and home delivery (1\%). Table 3

Table 3: Distribution of Neonatal sepsis based on place of delivery

\begin{tabular}{|c|c|c|c|}
\hline \multicolumn{2}{|c|}{ Sepsis } & No. of patients & $\mathbf{\%}$ \\
\hline \multirow{3}{*}{ Intramural } & BTGH & 34 & 34.0 \\
\cline { 2 - 4 } & STGH & 11 & 11.0 \\
\cline { 2 - 4 } & Sub Total & 45 & 45.0 \\
\hline \multirow{4}{*}{ Extramural } & Private hosp. & 29 & 29.0 \\
\cline { 2 - 4 } & Primary Health centre & 16 & 16.0 \\
\cline { 2 - 4 } & Govt. General Hosp & 9 & 9.0 \\
\cline { 2 - 4 } & Home Delivery & 1 & 1.0 \\
\cline { 2 - 4 } & Sub Total & 55 & 55.0 \\
\hline \multicolumn{2}{|c|}{ Grand Total } & 100 & 100.0 \\
\hline
\end{tabular}

In the study EOS were 59 (59.0\%) and LOS been 41 (41.0\%) patients. EOS more common than LOS. Table 4

Table 4: Distribution of Neonatal Sepsis based on age of onset

\begin{tabular}{|c|c|c|}
\hline Sepsis & No. of patients & \% \\
\hline EOS & 59 & 59.0 \\
\hline LOS & 41 & 41.0 \\
\hline Total & 100 & 100.0 \\
\hline
\end{tabular}

In our study most common presentation is tachypnea, followed by lethargy. Table 5

Table 5: Distribution of patients according to clinical presentation

\begin{tabular}{|c|c|c|c|}
\hline \multicolumn{2}{|c|}{ Sepsis } & No. of patients & $\%$ \\
\hline \multicolumn{2}{|c|}{ RDS } & 27 & 27.0 \\
\hline \multicolumn{2}{|c|}{ Lethargy } & 20 & 20.0 \\
\hline \multirow{4}{*}{ Preterm } & LGA & 6 & 6.0 \\
\hline & AGA & 21 & 21.0 \\
\hline & SGA & 3 & 3.0 \\
\hline & Total & 30 & 30.0 \\
\hline \multicolumn{2}{|c|}{ Poor feeding } & 7 & 8.0 \\
\hline \multicolumn{2}{|c|}{ Fever } & 6 & 6.0 \\
\hline \multicolumn{2}{|c|}{ Convulsions } & 5 & 5.0 \\
\hline \multicolumn{2}{|c|}{ Icterus } & 5 & 5.0 \\
\hline \multicolumn{2}{|c|}{ Abdominal Distension } & 3 & 3.0 \\
\hline \multicolumn{2}{|c|}{ Birth asphyxia } & 3 & 3.0 \\
\hline \multicolumn{2}{|c|}{ MAS } & 2 & 2.0 \\
\hline \multicolumn{2}{|c|}{ Excessive crying } & 2 & 2.0 \\
\hline \multicolumn{2}{|c|}{ Vomiting } & 2 & 2.0 \\
\hline \multicolumn{2}{|c|}{ Decreased activity } & 1 & 1.0 \\
\hline \multicolumn{2}{|c|}{ Excessive Frothing } & 1 & 1.0 \\
\hline \multicolumn{2}{|c|}{ H/0 Aspiration } & 1 & 1.0 \\
\hline \multicolumn{2}{|c|}{ Ruptured meningocele } & 1 & 1.0 \\
\hline \multicolumn{2}{|c|}{ Shallow Respiration } & 1 & 1.0 \\
\hline
\end{tabular}

\section{Discussion}

More than 30-80\% of neonates with proven infection become thrombocytopenic ${ }^{[8,9]}$. Bacterial, fungal and viral infections all have been associated with neonatal thrombocytopenia ${ }^{[10]}$.

Thrombocytopenia occurs in one-third of infants admitted in neonatal intensive care unit. Thrombocytopenia is frequently associated with mucosal bleeds and purpura.

Fungal sepsis is associated with greater degree of thrombocytopenia than is seen with gram positive or gram negative organisms and outcome in these neonates is poor [11].

MPV levels may increase in mild inflammation because of the raise of large platelets, or on the contrary, MPV levels may decrease in severe inflammation owing to the depletion of large platelets in inflammatory area ${ }^{[12]}$.

Destructive thrombocytopenia known to be associated with high MPV levels while low level of MPV is reported in hypo-proliferative thrombocytopenia ${ }^{[13]}$.

These observations indicate that MPV may be a negative acute phase reactant as well as a positive acute phase reactant and may show fluctuation in different phases of sepsis.

In our study we made an attempt to see association of platelet count and their indices in neonatal sepsis.

Comparison of Cases according to sex: Sex Incidence in our study was of male predominance. In our study Male/Female ratio is 1.8:1. Our study result is consistent with Woranart et al study which showed that males had higher incidence than female neonates ${ }^{[14]}$.

According to onset of sepsis: In our study EOS (59\%) is more common than LOS (41\%).

Antoniette B et al reported early onset of sepsis within 24 hours in $85 \%$ cases ${ }^{[15]}$.

Clinical presentation: In the present study Respiratory distress (27\%) and lethargy (20\%) were the commonest clinical presentation followed by Refusal of feeds (8\%), Fever (6\%), convulsions (5\%) and Icterus (5\%). Lethargy, refusal of feeds and respiratory difficulties were the commonest presenting combination of complaints. [16]. Ahsan Ahmad et al, reported that fever (46\%) was the most frequent symptoms, followed by respiratory difficulties (39\%), lethargy (37\%), refusal of feeds (33\%), jaundice (21\%) and convulsions (18\%) ${ }^{[17]}$. Shrestha P et al reported that commonest signs and symptoms were refusal of feeds (42.7\%), fever $(41.7 \%)$, jaundice $(41.7 \%)$, respiratory difficulties (29.2\%), convulsions (21.4\%) and lethargy $(14.6 \%)^{73}$. The above studies also reported similar presentation as in our study.

In our study 6 organisms was isolated and all these organisms were associated with some form of thrombocytopenia.

Among them gram negative sepsis (57\%) is more common than gram positive sepsis. In gram negative sepsis (57\%) 
most common organism is klebsiella pneumonia (24\%), $E$ coli (24\%) followed by pseudomonas (9\%). In gram positive organism (40\%) staphylococcus aureus (37\%) was the most common organism causing sepsis.

Parvez Rajnesh ${ }^{[18]}$ proved in their study that gram negative organisms causing sepsis were $54 \%$. In that most common were Klebsiella, followed by pseudomonas then acinetobacter and gram positive were $40 \%$, of which staphylococcus was most common followed by Enterococcus.

\section{Conclusion}

Neonatal sepsis has vague signs and symptoms, so high index of suspicion helps in arriving at early diagnosis and management of sepsis. Neonatal sepsis was common in males. Gram positive organisms were the predominant causative agents of septicaemia $40 \%$ as compared to gram negative organisms $57 \%$ and fungal sepsis $3 \%$. Staphylococcus aureus was the commonest organism responsible for thrombocytopenia.

\section{Acknowledgement}

I would like to express my profound gratitude to all the participants for their co-operation and for their immense faith they reposed in me.

\section{References}

1. Stoll BJ, Shane AL. Infections of the neonatal infant. Nelson Textbook of Pediatrics. 1st south Asia ed. Elsevier Publication, 914-5.

2. Israels SJ, Rand ML, Michaelson AD. Neonatal function, 2003, 29.

3. Nathan DG, orkin stuart H, A thomas haematology of infancy and childhood, 2003.

4. Murray NA, Roberts IA circulating megakaryocytes and their progenitors, 1996.

5. Wasiluk A. Thrombopoesis in healthy term newborns human fetal blood, 2005.

6. Forestier F, Daffos F, catherine $\mathrm{N}$, Renard $\mathrm{M}$, developmental hematopoeisis in normal human fetal blood.

7. Brown DR, Kutler D, Rai B, Chan T, Cohen M. Bacterial concentration and blood volume required for a positive blood culture. J Perinatol 1995;15(2):157-9.

8. Forsteier F, Daffos F, Galactéros F, Bardakjian J, Rainaut M, Beuzard Y et al. Haematological values of 163 normal foetuses between 18- 30 weeks of gestation. Pediatr Res 1986;20(4):342.

9. Laura A Stokowski. Neonatal thrombocytopenia evaluation and management. National association of neonatal nurses- 22 annual conference, 46.

10. Christensen RD, Henrey E, Weidmeier SE et al. Thrombocytopenia and neutropenia among ELBW neonates Hematological Report 2006;2(10):126-132.

11. Mhada TV, Fredrick F, Matee MI, Massawe A. Neonatal sepsis at Muhimbili National Hospital, Dar es Salaam, Tanzania; aetiology, antimicrobial sensitivity pattern and clinical outcome. BMC Public Health 2012;12:904.

12. Nelson RB 3rd, Kehl D. Electronically determined platelet indices in thrombocytopenic patients. Cancer. 1981;48(4):954-6.

13. Charoo BA, Iqbal JI, Iqbal Q, Mushtaq S, Bhat AW, Nawaz I. Nosocomial sepsis-induced late onset thrombocytopenia in a neonatal tertiary care unit: a prospective study. Hematol Oncol Stem Cell Ther 2009;2(2):349-353.

14. Ratanakorn W, Srijariya W, Chamnanvanakij S, Saengaroon P. Incidence of neonatal infection in newborn infants with maternal history of premature rupture of membranes (PROM) for 18 hours or longer by using pharmong Kutklar Hospital clinical practice guide lines (CPG). J Med Assoc. Thai 2005;8(7):973.

15. Antoniette BWM. Flora DIP. Clinical Correlation of Neonatal and Maternal Hematological Parameters As Predictors of Neonatal Sepsis. PIDSP Journal 2005;9(2):36-43.

16. Singh M, Narang A, Bhakoo ON. "Evaluation of a Sepsis Screen in the diagnosis of neonatal sepsis. Indian Pediatr 1987;24(1)39-43.

17. Ahmad A, Hussain W, Lamichhane A, Aslam M, Riaz L. Use of Antibiotics in Neonatal Sepsis at Neonatal Unit of A Tertiary care Hospital. Pak Paed J 2011;35(1):3-7.

18. Ahmad P, Kaith R, Gattoo I, Najar BA, Hussain SQ. Thrombocytopenia as a predictor of neonatal sepsis in very low birth weight babies. Indian Journal of Neonatal Medicine and Research 2015;3(3):7-13. 